Mansoura University

Faculty of Education

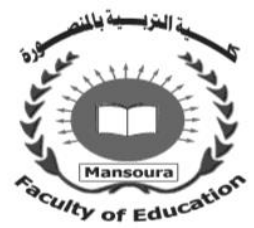

Efficacité de l'utilisation de la stratégie de résumé sur le développement des compétences de la compréhension des textes chez les étudiants du cycle secondaire des écoles de langues

\author{
By \\ Ahmed Mohamed Chendi \\ Sous la direction de
}

Superuised by

Dr. Rifqi Ibrahim SOLEIMAN Dr. Wadha Mansour ALAHWAL

Professeur-adjoint des curricula Maître de conférences des curricula et didactique de la langue française

Faculté de Pédagogie et didactique de la langue française

Université de Mansourah

Faculté de Pédagogie

Université de Mansourah

Journal Of The Faculty Of Education-Mansoura University

No. 112 - Oct .2020 


\title{
Efficacité de l'utilisation de la stratégie de résumé sur le développement des compétences de la compréhension des textes chez les étudiants du cycle secondaire des écoles de langues
}

\author{
Ahmed Mohamed Chendi
}

\section{1- Introduction:}

La langue joue un rôle très important dans la vie des individus et des sociétés et l'homme utilise la langue pour exprimer et communiquer ses besoins, ses ambitions, ses rêves et ses sentiments : joie, malheur, surprise, admiration.

Les langues étrangères jouent un rôle primordial à ouvrir les horizons des apprenants sur le monde entier. C'est pour cela qu'on accorde à l'enseignement des langues étrangères une grande importance afin de rendre les étudiants capables de communiquer et de s'adapter aux nouveaux changements dans tous les domaines.

La langue est un moyen de communication entre les peuples. Selon Soleiman (1996: 2) elle ".... est donc un moyen de communication qui comporte 4 compétences langagière: Entendre et Comprendre, Parler, Lire et Ecrire. L'apprentissage/l'enseignement d'une langue étrangère doit donc prendre en considération ces 4 compétences comme unité intégrante sans négliger l'une ou l'autre".

Pour bien s'exprimer, on a donc besoin de développer les quatre compétences langagières: écouter et parler qui traitent la langue exprimée au moyen d'un support non visuel; écrire et lire qui traitent la langue exprimée au moyen d'un support visuel. En effet, l'enseignement/ apprentissage d'une langue étrangère doit prendre en considération ces quatre compétences comme une unité intégrale sans négliger l'une ou l'autre.

La compréhension des textes est le but essentiel de la lecture et constitue un processus interactif qui met en relation un lecteur et un texte dans un contexte. Dans ce processus, le lecteur crée le sens en se servant à la fois du texte, de ses propres connaissances et de son intention de lecture. (Giasson, 2000: 5). 
Le résumé consiste à traiter les informations d'un texte pour garder que les points les plus importants. Il faut éviter les informations secondaires, répétitives ou semblables, tout en représentant l'opinion de l'auteur. Cette stratégie de lecture est très importante, puisqu'en traitant l'information, nous la gardons en mémoire. On peut réemployer la technique du résumé pour mieux se faire comprendre et mieux exprimer sa pensée. Que ce soit pour se préparer avant un examen ou durant n'importe quelle activité de lecture, cette stratégie sera utile pour la vie.

Pour faire un bon résumé, il faut avoir en tête trois objectifs : La compréhension des textes en lecture est une compétence linguistique très importante car elle aide à développer chez l'apprenant la capacité à réfléchir et à comprendre. On lit donc pour développer notre personnalité, pour élargir ses expériences et ses connaissances et pour savoir ce qui se passe autour de lui.

Le chercheur utilise la stratégie de résumé pour développer certaines competences de la comprehension des textes qui permet aux étudiants de comprendre de façon active pendant la lecture.Le résumé a pour but de rendre compte des idées les plus importantes d'un texte.

Pour faire un résumé, il faut suivre trois étapes, l'élimination des informations secondaires et celles qui se répètent, la substitution qui vise à remplacer une liste de mots par un seul mot qui les englobe tous, la sélection qui permet de sélectionner l'énoncé qui comprend l'idée principale du texte. (Site d'internet no.35)

1. Le résumé doit garder et contenir l'essentiel des informations en n'omettant pas l'opinion de l'auteur

2. Les informations secondaires et qui se répètent doivent être éliminées.

Le but n'est pas d'écrire un texte aussi long que l'original mais bien d'aller droit au but. Bien que l'information contenue dans le résumé est la même que dans le texte original, il faut apprendre à la trier pour éviter la redondance.

3. Le résumé doit être adapté au destinataire

Il existe trois étapes aident à écrire un bon résumé: l'élimination, la substitution et la sélection.

\section{L'élimination}

Éliminer les informations secondaires et celles qui se répètent. Ils ne faut pas nécessairement garder les détails intéressants, mais garder ceux qui sont utiles. Il ne faut pas répéter ce qui a déjà été dit. Sans éliminer l'information essentielle, on peut réécrire cette phrase pour retirer le superflu. 


\section{La substitution}

Ce procédé consiste à remplacer une liste de mots par un seul mot qui les englobe tous.

\section{La sélection}

Ce procédé sert à sélectionner la phrase qui contient l'idée principale du texte et la reformuler en utilisant le procédé de l'élimination..(Site d'internet $\mathrm{N}^{\mathrm{o}} 36$ )

\section{Sensibilisation au problème de la recherche}

Malgré l'importance de la compréhension des textes comme moyen d'apprentissage/d'enseignement de toute langue, ainsi d'adaptation à la société, il y a beaucoup de difficultés dans la compréhension des textes.

Dans cette perspective, on constate que le développement des compétences de la compréhension des textes a constitué l'objectif de plusieurs études effectuées dans le domaine de la didactique du FLE comme: L'étude de Makhlouf (2001), Baier (2005), Elsherbini (2007), Bahie El Dine (2008), Milad (2008), Ramadan (2008) et Sabri (2009).Fouad (2014), Abdel-Aal (2016), Hamed (2019).

Le chercheur consulte ces études antérieures pour confirmer l'existence du problème de la recherche du fait qu'elles nous aident à déterminer les compétences nécessaires à l'amélioration la compréhension des textes.

\section{Problématique de la recherche}

La problématique de l'étude réside dans la faiblesse des compétences de la compréhension des textes chez les étudiants du cycle secondaire des écoles de langues. Ainsi la problématique de la recherche se résume dans les questions suivantes:

- Quelle est l'efficacité de l'emploi de la stratégie de résumé sur le développement de certaines compétences de la compréhension des textes?

\section{Objectif de la recherche}

La recherche actuelle vise à :

- Préciser l'efficacité de la stratégie de résumé sur le développement de certaines compétences de la compréhension des textes chez les étudiants du cycle secondaire des écoles de langue.

\section{Méthodologie de la recherche}

Le chercheur va utiliser pendant la recherche actuelle la méthode descriptive et la méthode quasi-expérimentale.

\section{Outils de la recherche}


- Test de mesure des compétences de la compréhension des textes chez les étudiants du cycle secondaire des écoles de langue.

\section{Hypothèse de de la recherche}

- Il y a des différences significatives statistiquement entre la moyenne des notes des étudiants du groupe témoin et ceux du groupe expérimental au post test des compétences de la compréhension des textes en faveur du groupe expérimental.

- Il y a des différences significatives statistiquement entre la moyenne des notes des étudiants du groupe expérimental au pré/post test des compétences de la compréhension des textes en faveur du post/test.

\section{Délimites de de la recherche}

Cette recherche se limite à:

1. Un échantillon d'étudiants du cycle secondaire des écoles de langues françaises.

2. Certaines compétences de la compréhension des textes nécessaires aux étudiants du cycle secondaire des écoles de langues: école de Mansourah expérimentale de langues.

3. Deux unités de la méthode enseignée intitulée "club @ dos 2" reformulées selon la stratégie de résumé.

\section{Procédures de de la recherche:}

Pour répondre à la questions de la recherche, le chercheur a poursuivi les étapes suivantes:

- Passer en revue les études théoriques et les recherches antérieures qui se rapportent à la recherche actuelle.

- Préparer un test des compétences de la compréhension des textes nécessaires aux étudiants du cycle secondaire des écoles de langue.

- Présenter le test de la recherche au jury pour vérifier sa validité apparente.

- Modifier le test d'après les points de vue du jury.

- Standardiser le test (fidélité et validité).

- Choisir l'échantillon de la recherche et le diviser en deux groupes: expérimental et témoin.

- Appliquer le pré-test en vue de diagnostiquer les compétences de la compréhension des textes chez l'échantillon de la recherche.

- Choisir et reformuler des deux unités no. (1 et 2) de la méthode utilisée intitulée : "club @ dos 2"

- Enseigner les deux unités reformulées selon la stratégie de résumé au groupe expérimental. 
- Appliquer le post-test sur les deux groupes de la recherche.

- Analyser statiquement les résultats de la recherche.

- Interpréter les résultats de la recherche.

- Présenter les recommandations et les suggestions de la recherche.

\section{Terminologies de la recherche}

\section{La compréhension des textes}

Aksan et Kisac (2009), cité par Mohammed (2015) définissent la compréhension des textes par un processus mental utilisé par le lecteur afin d'extraire les idées et les informations, d'entendre les aspects affectifs que l'auteur vise à transmettre à un lecteur tout en employant de stratégies de lecture qui lui aide à éviter le malentendu.

Foulin et Toczek (2006: 41), présentent une définition de la compréhension des textes en disant " elle est une activité cognitive complexe qui mobilise des connaissances déclaratives, des mécanismes de traitement cognitif, des métaconnaissances et des ressources attentionnelles.

\section{La stratégie de résumé}

Selon Vacca (2002: 307), un résumé consiste à « Le concision d'un texte en ses points principaux ».

\section{Cadre théorique}

\subsection{Première axe du cadre théorique: la compréhension des textes}

\subsubsection{Notion de la lecture}

La lecture est un moyen important pour élargir les connaissances du lecteur.

D'un point de vue psycholinguistique, la lecture peut être considérée comme un processus qui comprend trois phases : anticipation (production d'une hypothèse sur le texte, le contrôle (vérification de I'hypothèse) et mémorisation (stockage de l'information acquise dans la méoire. (Déscôtés, M., 1999)

Selon Nilsson (2006: 4), cité par Abdelaal (2014: 19), la lecture du texte en langue étrangère, ne se limite pas à la compréhension du texte lu mais suscite également de diverses réflexions et des réactions de la part du lecteur. Nous considérerons, néanmoins, que la lecture implique des processus qui contribuent à divers degrés à une compréhension du texte lu. Elle dépendrait des connaissances antérieures du lecteur et notamment des connaissances linguistiques.

Pour Sobhi (2010: 58-59), la lecture ne signifie pas seulement la capacité à reconnaître les lettres, les mots et la prononciation des sons, mais aussi elle consiste à comprendre le sens des mots et des phrases. Elle est 
conçue comme activité cognitive de prise et de traitement de l'information pendant laquelle le lecteur (identifie des signes) et construit le sens.

\subsection{2. Étapes de la lecture}

- D'après Martin (1999: 34), il y a quatre étapes de la lecture:

- L'avant lecture du texte: met en jeu les connaissances et les représentations initiales des apprenants.

- La lecture découverte ou balayage: est l'activité la plus évidente et qui correspond aux premières réactions du lecteur.

- La lecture sélective et organisée: est toujours une relecture soucieuse pour construire le sens.

- Les nouvelles perspectives de lecture du texte: d'autres textes permettent de sortir du carcan d'une démarche rigoureuse, de faire le point sur les acquis en les mettant au service de projets plus libres et plus personnels.

\subsubsection{Notion de la compréhension des textes}

Le but du processus de la lecture est la compréhension, Elle permet à l'étudiant de relier une information à un modèle mental.

D'autre part, Giasson (2004: 22) voit que le lecteur construit le sens du texte à partir de l'information implicite et explicite du texte et de ses connaissances antérieures.

La compréhension, comme le cite Robert (2002), vient de comprehensio, mot issu de "compréhension" qui signifie en latin, au sens propre, "saisir, enfermer" 'saisir par intélligence, embrasser par la pensée.

Selon le dictionnaire de didactique du français langue étrangère et seconde, la compréhension écrite, est l'aptitude résultant de la mise en œuvre de processus cognitifs, qui permet à l'apprenant d'accéder au sens d'un texte qu'il lit. La lecture conduit, en fonction de l'objectif poursuivi, à percevoir soit de manière exhaustive tous les éléments du texte, soit de manière sélective certains de ces éléments du texte, pour mener à une compréhension qui peut être globale ou détaillée. (Cuq, J., p., 2003).

De sa part, Dejamanchai (1999) souligne que la compréhension écrite est conçue comme une opération active de la construction du sens dans laquelle le lecteur relie les informations nouvelles apportées dans le texte aves ses connaissances préconstruites.

\subsubsection{Types de textes}

Dans son analyse des schémas textuels prototypiques, Adam (1992: 128) précise les types suivants:.

\section{- Texte explicatif informatif}


C'est par le texte informatif, qu'on peut renseigner sur un sujet donné. Le texte informatif, en revanche, ne vise pas à établir une conclusion: il transmet des données, certes organisées, hiérarchisées, mais pas à des fins démonstratives. Il ne s'agit pas, en principe, d'influencer l'auditoire, de le conduire à telle ou telle conclusion, de justifier un problème qui serait posé.

\section{- Texte descriptif}

Le texte descriptif se construit selon, Jeandillou (1997: 149), autour d'une unité thématique (objet, lieu ou individu) dont sont pris en considération l'aspect général, les parties constitutives ou la situation dans le temps et l'espace. Le thème choisi peut en outre être appréhendé, par le biais de métaphores ou de comparaisons, grâce aux similitudes qu'il présente avec d'autres éléments.

On peut dire que le texte descriptif est un type de texte qui sert à décrire un objet, un personnage ou un lieu.

\section{- Texte argumentatif}

On peut dire que, le texte argumentatif vise à convaincre le lecteur autour d'une idée ou d'un avis.

Selon Boissinot, cité par Lafarge (1996: 21-22), la structure du texte argumentatif est véritablement dialogique: elle s'organise en deux pôles, ceux des thèses qui s'affrontent: thèse proposée et thèse refusée. Il s'agit pour le texte argumentatif de faire passer son auditoire, comme un jury dans un procès d'une thèse à l'autre ou plus simplement, de faire affronter deux pôles d'énonciation, les tenants de la thèse proposée, les tenants de la thèse refusée.

\section{- Texte narratif}

Ce type qui l'axe principal de la recherche actuelle, On le définit comme une suite d'actions ou d'événements réels ou imaginaires, à travers le temps, avec des situations plus ou moins stables et durables.

D'autre part, le récit peut suivre une progression différente de celle qu'impose la chronologie. D'où les phénomènes d'ellipse (avec occultation de certains faits), d'analepsie (en cas de retour en arrière) et de prolepse (en cas d'annonce anticipée de faits ultérieurs). Le champ narratif se caractérise non par sa conformité avec ce qu'il représente mais par les décalages qu'il est susceptible d'instaurer. (Jeandillou, 1997: 156).

\section{1.5. Processus de la compréhension du texte}

Selon le propos d'Elsayed (1996), la compréhension des textes comprend quatre processus essentiels:

1- Le processus lexical: C'est l'opération dans laquelle l'étudiant feuillette sa mémoire pour reconnaître les mots. 
2- Le processus syntaxique: C'est la reconnaissance des structures linguistiques et grammaticales.

3- Le processus sémantique : C'est la reconnaissance des sens des mots et des phrases.

4- Le processus pragmatique : C'est la capacité de communiquer avec les autres dans de nouvelles situations

Grabe (1991) démontre que la lecture est une combinaison de différents processus, elle est :

- Un processus rapide, dont le traitement de l'information dans la mémoire durant la compréhension d'un texte doit se caractériser par une vitesse convenable.

- Un processus flexible, dans la situation de lecture, le lecteur emploie une variété de stratégies pour comprendre ce qu'il lit d'une façon efficace.

- Un processus interactif, au cours de la lecture, la mémoire de travail du lecteur est constamment engagée dans une variété de processus qui constitue les tentatives mises en jeu pour reconstruire les intentions de l'auteur.

- Un processus stratégique, dont le processus de la compréhension doit être stratégique pour que le lecteur puisse gérer de nombreuses habiletés, déterminer les difficultés de traitement et prendre des décisions dans sa lecture.

- Un processus d'évaluation, il s'agit du lecteur qui doit décider si les informations apportées dans le texte qu'il lit sont intéressantes et pertinentes à son objectif et à ses motivations.

- Un processus d'apprentissage, les étudiants apprennent des nouvelles informations concernants plusieures matières à travers la lecture.

- Un processus de langue avant d'être un processus de raisonnement, le lecteur avant de pouvoir interpréter et comprendre un texte, il doit se familiariser linguistiquement avec lui.

\subsubsection{Difficultés de la compréhension des textes}

Giasson (2000: 1) déclare les facteurs qui influent sur la compréhension des trois variables comme suit:

\section{- Niveau 1}

Pour comprendre le texte qu'il est en train de lire, le lecteur doit avoir un intérêt à le lire, il doit aussi avoir des connaissances sur la langue et sur le sujet du texte.

Simultanément, il doit mettre en œuvre les cinq habiletés de la lecture: 
- Microprocessus: déchiffrage, identification des mots, propositions, phrases.

- Intégration: les connecteurs; lien entre les propositions et les phrases.

- Macroprocessus: idée principale, résumé, texte en tant qu'un tout cohérent.

- Élaboration: dépassement du texte, appropriation personnelle du texte.

- Métacognition: gestion de la compréhension qui permet les ajustements du lecteur.

\section{- Niveau 2}

Pour comprendre, le lecteur a besoin de représentations du texte, il doit savoir à quel type de texte il va avoir à faire, il doit connaître les intentions de l'auteur (Veut-il nous persuader, veut-il nous informer, veut-il nous distraire?), il doit saisir comment l'auteur a organisé ses idées (la forme) et ce que l'auteur veut nous transmettre (le contenu). La forme et le contenu sont imbriqués.

\section{- Niveau 3}

Le contexte de lecture correspond aux conditions dans lesquelles se trouve le lecteur au moment où il entre en contact avec le texte. Quelle est l'intention du lecteur? $\rightarrow$ que va-t- il chercher dans sa lecture? Dans quel contexte social se fait la lecture? $\rightarrow$ devant un public, en interaction avec un pair, ... etc. Le contexte physique joue également $\rightarrow$ bruit, qualité du document, confort de lecture.

\section{Deuxième axe du cadre théorique; la stratégie de résumé}

\subsection{Notion de la stratégie de résumé}

Un résumé est un «abrégé ou un condensé généralement présenté à la fin d'un volume, d'un article, d'une communication orale, d'un événement ou d'un phénomène, dans un but de récapitulation et parfois de conclusion »(Legendre 1993: 1122).

\subsection{Bases du résumé}

Il faut suivre les bases suivantes pour faire un bon résumé comme suit: - Élimination des données secondaires répétées.

- Substitution d'une liste des faits par un terme englobant.

- Sélection et formulation de la phrase qui contient l'idée principale.

\section{3. Étapes de la stratégie de résumé}

Weiner et Bazerman (1997: 393) determinent les étapes suivantes:

- Lire tout le texte.

- Extraire les idées principales.

- Marquer l'idée principale de chaque paragraphe.

- Reformuler idées à partir des phrases pour présenter les liens entre eux. 
- Garder les informations importantes et éviter de répéter les mots inutiles.

- Organiser et présenter les idées pour expliquer le sens du paragraphe

\section{Résultats de la recherche}

Cette recherche vise à vérifier l'efficacité de la stratégie de résumé pour le développement des compétences de la compréhension des textes chez les étudiants des écoles de langues françaises. Pour cela cette recherche comprend :

- Il y a des différences significatives statistiquement entre la moyenne des notes des étudiants du groupe témoin et ceux du groupe expérimental au post test des compétences de la compréhension des textes en faveur du groupe expérimental.

- Il y a des différences significatives statistiquement entre la moyenne des notes des étudiants du groupe expérimental au pré/post test des compétences de la compréhension des textes en faveur du post/test.

Afin de vérifier la première hypothèse de la recherche qui consiste à «l'existence des différences statistiquement significatives au niveau de $P(\leq \mathbf{0 . 0 5})$ entre la moyenne de notes des étudiants de deux groupes témoin et expérimental au post test des compétences de la compréhension des textes en faveur du groupe expérimental». Pour vérifier cette hypothèse, le chercheur a utilisé le "Test $\mathrm{T}$ " pour la signification des différences entre la moyenne de notes des échantillons indépendants.

Pour cela, le chercheur a calculé : la moyenne, l'écarte type des notes des étudiantes au post-test des compétences de la compréhension des textes. Ainsi, le chercheur a calculé la valeur de " $\mathrm{T}$ " correspondant à la différence des deux moyennes et il a déterminé le niveau de la signification correspondant la valeur de "Test $\mathrm{T}$ ". Le tableau suivant montre ces résultats.

\section{Tableau No (1)}

"Test $\mathbf{T}$ " de la signification des différences entre la moyenne de notes des étudiantes des deux groupes (témoin et expérimental) au post-test de mesure des compétences de la compréhension des textes

\begin{tabular}{|c|c|c|c|c|c|c|c|}
\hline Compétences & Groupes & $\mathbf{N}$ & M. & E. T. & D. L. & T. & S. \\
\hline \multirow{2}{*}{ 1) La prédiction } & Témoin & 32 & 5,8125 & 1,8217 & \multirow{2}{*}{62} & \multirow{2}{*}{5,1195} & \multirow{2}{*}{0,05} \\
\hline & Expérimental & 32 & 8,2500 & 1,9838 & & & \\
\hline \multirow{2}{*}{ 2) La clarification } & Témoin & 32 & 5,18875 & 1,6152 & \multirow{2}{*}{62} & \multirow{2}{*}{8,4658} & \multirow{2}{*}{0,05} \\
\hline & Expérimental & 32 & 8,5938 & 1,6036 & & & \\
\hline \multirow{2}{*}{ 3) Le questionnement } & Témoin & 32 & 5,8125 & 1,8568 & \multirow{2}{*}{62} & \multirow{2}{*}{6,5402} & \multirow{2}{*}{0,05} \\
\hline & Expérimental & $\overline{32}$ & 8,8438 & 1,8511 & & & \\
\hline \multirow{2}{*}{ 4) Le résumé } & Témoin & 32 & 2,9686 & 0,4004 & \multirow{2}{*}{62} & \multirow{2}{*}{15,5613} & \multirow{2}{*}{0,05} \\
\hline & Expérimental & $\overline{32}$ & 6,8750 & 1,3619 & & & \\
\hline \multirow{2}{*}{ Total } & Témoin & 32 & 19,7813 & 2,4195 & \multirow[b]{2}{*}{62} & \multirow{2}{*}{18,5029} & \multirow{2}{*}{0,05} \\
\hline & Expérimental & $\overline{32}$ & 32,5625 & 3,0684 & & & \\
\hline
\end{tabular}




\section{Légende :}
N. : Nombre d'étudiantes
D. L. : Degré de liberté
M. : Moyenne des Notes
T. : Valeur de "Test T"
E. T. : Écart type
S. : Signification

Les chiffres du tableau no. (1) montre qu'il y a des différences statistiquement significatives au niveau de $\mathrm{P}(\leq 0.05)$ entre la moyenne de notes des étudiants de deux groupes témoin et expérimental au post test des compétences de la compréhension des textes en faveur du groupe expérimental.

La dispersion de notes des étudiantes du groupe expérimental au posttest en ce qui concerne les compétences de la compréhension des textes indique que leur niveau est plus élevé. Cela prouve que le niveau des étudiantes du groupe expérimental est plus élevé que celui du groupe témoin au post-test. Donc, cette première hypothèse s'est réalisée et confirmée.

Afin de vérifier la deuxième hypothèse de la recherche qui consiste à « l'existence des différences statistiquement significatives au niveau de $P(\leq 0.05)$ entre la moyenne des notes des étudiants du groupe expérimental au pré/post tests des compétences de la compréhension des textes en faveur du post/test». Le chercheur a eu recours au test « $T$ » pour la signification des différences entre la moyenne des notes des échantillons dépendants.

Pour cela, le chercheur a calculé : la moyenne, écarte type pour les notes des étudiantes du groupe expérimental au pré-post tests des compétences de la compréhension des textes. Ainsi, il a ensuite calculé la valeur de « $\mathrm{T} »$ correspondant à la différence des deux moyennes et a déterminé le niveau de la signification correspondant à cette valeur de « $\mathrm{T} »$. Le tableau suivant présente ces résultats du test $« T$ $T$ :

Tableau No (2)

"Test T" de la signification des différences entre la moyenne de notes des étudiantes du groupe expérimental aux pré/post-tests des

compétences de la compréhension des textes

\begin{tabular}{|c|c|c|c|c|c|c|c|}
\hline Compétences & Test & $\mathbf{N}$ & M. & E. T. & D. L. & T. & S. \\
\hline \multirow{2}{*}{ La prédiction } & Pré-test & 32 & 5,0625 & 2,2024 & \multirow{2}{*}{62} & \multirow{2}{*}{6,0334} & \multirow{2}{*}{0,05} \\
\hline & post-test & 32 & 8,2500 & 1,4838 & & & \\
\hline \multirow{2}{*}{$\begin{array}{l}2) \quad \text { La } \\
\text { clarification }\end{array}$} & Pré-test & 32 & 5,5625 & 1,8997 & \multirow{2}{*}{62} & \multirow{2}{*}{6,9452} & \multirow{2}{*}{0,05} \\
\hline & post-test & 32 & 8,5938 & 1,6036 & & & \\
\hline \multirow{2}{*}{$\begin{array}{cc}\text { 3) } & \text { Le } \\
\text { questionnement }\end{array}$} & Pré-test & 32 & 6,3438 & 1,6186 & \multirow{2}{*}{62} & \multirow{2}{*}{5,6532} & \multirow{2}{*}{0,05} \\
\hline & post-test & 32 & 8,8438 & 1,8511 & & & \\
\hline \multirow{2}{*}{ Le résumé } & Pré-test & 32 & 2,4357 & 1,1053 & \multirow{2}{*}{62} & \multirow{2}{*}{16,2609} & \multirow{2}{*}{0,05} \\
\hline & post-test & 32 & 6,8750 & 1,3619 & & & \\
\hline \multirow{2}{*}{ Total } & Pré-test & 32 & 19,4063 & 2,6864 & \multirow{2}{*}{62} & \multirow{2}{*}{16,1023} & \multirow{2}{*}{0,05} \\
\hline & post-test & 32 & 32,5625 & 3,0684 & & & \\
\hline
\end{tabular}




\section{Légende :}
N. : Nombre d'étudiantes
D. L. : Degré de liberté
M. : Moyenne des Notes
T. : Valeur de "Test T"
E. T. : Écart type
S. : Signification

Le tableau no. (2) indique qu'il y a des différences statistiquement significatives au niveau de $\mathrm{P}(\leq 0.05)$ entre la moyenne de notes des étudiantes du groupe expérimental aux pré/post-tests en ce qui concerne les compétences de la compréhension des textes en faveur du post-test.

Le niveau des étudiantes du groupe expérimental est plus élevé au post-test en ce qui concerne les compétences la compréhension des textes que celui du pré-test.

La dispersion de notes des étudiantes du groupe expérimental au posttest en ce qui concerne les compétences de la compréhension des textes indique que leur niveau est plus élevé et que leurs notes sont homogènes après l'étude de deux unités reformulées par l'utilisation de la stratégie de résumé.

Les résultats précédents indiquent la validation de la deuxième hypothèse de la recherche et également l'efficacité de la stratégie de résumé dans le but de développer les compétences de la compréhension des textes des étudiants du cycle secondaire des écoles de langues françaises.

Après avoir vérifié les deux hypothèses de la recherche, le chercheur présente dans les lignes qui suivent la manière par laquelle il procédé afin de vérifier la taille de l'efficacité de la stratégie de résumé sur le développement des compétences de la compréhension des textes des étudiants du cycle secondaire des écoles de langues françaises.

Pour ce faire, le chercheur a calculé la valeur de « $\mathrm{T} »$ et la valeur de $« \eta 2 »$ pour, comme il est déjà cité, déterminer la taille de l'effet(D. E.)_de la variable indépendante qui est la stratégie de résumé sur le développement des compétences de la compréhension des textes chez les étudiants.

\section{Le tableau suivant montre ces résultats:}

Tableau (3)

Degré de l'effet de l'utilisation de la stratégie de résumé

\begin{tabular}{|c|c|c|}
\hline Compétences & $\boldsymbol{\eta}^{2}$ & D. E. \\
\hline La prediction & 0,5401 & Élevé \\
\hline La clarification & 0,6088 & Élevé \\
\hline Le questionnement & 0,5076 & Élevé \\
\hline Le résumé & 0,8951 & Élevé \\
\hline Total & 0,8932 & Élevé \\
\hline
\end{tabular}

Légende :

$\boldsymbol{\eta}^{2}$ : Eta Carré.

D. E : Degré de l'effet 
Les chiffres du tableau ci-dessus montre que les valeurs de $(\eta 2)$ sont pour les compétences de la compréhension des textes entre $(0,5076)$ et $(0,8951)$, tandis que la valeur de $(\eta 2)$ pour le total de ces compétences est $(0,8932)$. Cela signifie que le degré de l'effet de l'utilisation de la stratégie de résumé est élevé.

\section{Interprétation des résultats de la recherche}

À la lueur des résultats obtenus du post-test à partir l'analyse des copies des étudiants après l'enseignement de deux unités reformulées, il est évident qu'il y a un développement remarquable dans la performance des étudiants du groupe expérimental en ce qui concerne les compétences de la compréhension des textes. Autrement dit, le chercheur a trouvé que le nombre total des compétences de la compréhension des textes chez les étudiants du groupe expérimental a été élevé au post-test, alors que ces compétences restent diminuées chez leurs collègues du groupe témoin.

\section{Recommandations de la recherche}

À la lumière les résultats de cette recherche, le chercheur présente les recommandations suivantes:

- La nécessité de donner la priorité aux compétences de la compréhension des textes dans l'enseignement des langues étrangères.

- Motiver les apprenants d'être au centre du processus de l'apprentissage et non seulement un récepteur d'information.

- La nécessité de l'utilisation des stratégies interactives qui encouragent les étudiants à apprendre dans un milieu favorable à la coopération, à la présentation et à la réflexion.

- Il faut que les enseignants prennent en considération la conscience métalinguistique des apprenants pour améliorer l'enseignement de la compréhension des textes de ces apprenants.

- La nécessité de constituer les programmes du cycle secondaire d'une façon permettant aux étudiants dès le début de l'apprentissage de la langue française à pratiquer le français comme une langue de communication en et hors classe avec leurs collègues.

- L'enseignement doit s'intéresser à développer les compétences sociales à côté des compétences cognitives et linguistiques.

- La nécessité d'aider les étudiants à construire des liens entre les nouvelles connaissances et ses connaissances antérieures.

\section{Suggestion de la recherche}

Cette recherche est considérée comme un point de départ qui attire l'attention d'autres chercheurs pour voir les inconvénients dans l'enseignement de la langue française et surtout au cycle secondaire en ce 
qui concerne ses trois pôles: l'apprenant, l'enseignant et les méthodes d'enseignement.

\section{Bibliographie}

1. Abdel-Aal, M. (2016). Efficacité de la stratégie de l'enseignement réciproque pour le développement des compétences de la compréhension des textes lus et l'attitude envers la langue française chez les étudiants du cycle secondaire d'Al Azhar. Thèse de magistère. Faculté de pédagogie. Université de Mansourah.

2. Afifi, A. (2018). Efficacité d'un programme basé sur l'enseignement différencié sur le développement des compétences de la compréhension en lecture chez les étudiants de la section de français. Thèse de Magistère Faculté de Pédagogie. Université d'Al Azhar.

3. Alsafy, C. (2010). Efficacité d'un programme proposé basé sur l'approche du processus de l'écriture pour le développement de quelques compétences de la production écrite et les attitudes des élèves du cycle secondaire. Thèse de Doctorat, Faculté de jeunes fillesUniversité d'Ain Chams.

4. . Bahie El Dine, M. (2008). Efficacité d'un programme propose en vue de développer les compétences de la lecture silencieuse et de la pensée critique chez les futurs enseignants de français. Thèse de Magistère. Faculté de jeunes filles. Université d'Ain chams

5. Baier, R. (2005) Reading comprehension and reading strategies. Master of Education Degree in Education. The Graduate School University of Wisconsin-Stout.

6. Cartier, S. et Théorêt, M. (2004). L'enseignement des strategies d'apprentissage par la lecture. Albums, Paris: Hachette.

7. Ciborowski, J. (1998). Textbook and the Students Who CanÕt Read Them, Cambridge, Brookline Books, chap. 4.

8. Cuq, J.-P. (2003). Dictionnaire de didactique du français langue étrangère et seconde. Paris: CLE International.

9. Dejarnonchai, S. (1999). Importance relative des variables liées au lecteur, Au texte et à l'interaction lecteur/texte sur la compréhension écrite en français langue étrangère d'élèves thai de fin du secondaire. Thèse de doctorat. Faculté des études supérieures. Université Laval.

10. Déscôtés, M. (1999). La lecture méthodique.Toulouse: CRDP de Midi- Pyrénées : Delagrave.

11.El-Chahat, G. (2000). Efficacité du programme proposé pour le développement des compétences de lecture d'étude chez les étudiants 
des départements de français des facultés de pédagogie. Thèse de Doctorat. Faculté de pédagogie. Université de Mansourah.

12. Elsherbini, I. (2007). The effectiveness of SQ3R strategy in developing the reading comprehension skills of EFL secondary students at AlAzhar. Master. Faculty of education. Mansoura University.

13. Fouad, R. (2014). Efficacité de l'emploi de la stratégie des cartes sémantiques sur le développement des compétences de la compréhension lecturale chez les étudiants de la faculté de pédagogiesection de français. Thèse de Magistère. Faculté de pédagogie. Université de Mansourah.

14. Foulin, J. N. et Toczek, M. (2006). Psychologie de l'enseignement. Armand Colin.

15. Giasson, J. (2000). La compréhension en lecture. Paris: De Boeck.

16. Giasson, J. (2004). La lecture de la théorie à la pratique. Paris: De Boeck.

17. Giasson, J. (2013). La lecture: apprentissage et difficulté. Bruxelles: De Boeck.

18. Hafez, H. (2007). Programme proposé pour remédier à quelques difficultés de lecture chez les élèves de la deuxième année primaire. Revue de la faculté de Pédagogie. Université d'Ain Chams. 13 (2), 243-288.

19. Hamed, D. (2019). Efficacité de l'utilisation de la stratégie «S V A » sur le développement des compétences de la compréhension des textes narratifs en français chez les étudiants du cycle secondaire des écoles de langue. Thèse de Magistère. Faculté de Pédagogie - Université de Mansourah.

20. Legendre, R. (1993). Dictionnaire actuel de l'éducation, (2e éd.). Montréal, Guérin.

21. Makhlouf, M. (2001). Approches textuelles et didactique du texte. Mémoire de Magistère. Faculté des lettres et langues - Université d'Alger.

22. Maring, G. H. et Furman, G. (1985). Seven "Whole Class" Strategies to Help Mainstreamed Young People Read and Listen Better in Content Areas Classes. Journal of Reading, 28 (8), 694-700.

23. Milad, A. (2008). L'influence de l'apprentissage de la langue anglaise sur l'acquisition d'une compétence de la lecture en français (langue étrangère seconde) au cycle secondaire. Thèse de Magistère. Institut d'Etudes Pédagogiques.

24. Martin, M. (1999). Jeux pour lire : apprendre à lire-écrire avec des 
25. Mohammed, N. (2015). Efficacité d'emploi des activités langagières basées sur l'approche constructiviste pour développer quelques Compétences de la Compréhension Écrite chez les Étudiants du Cycle Secondaire. Thèse de magistère. Université du Fayoum.

26. Pierre, R. (2003). L'enseignement de la lecture au Québec de 1980 à 2000: fondements historiques, épistémologiques et scientifiques. Revue des sciences de l'éducation.

27. . Ramadan, A. (2008). Efficacité d'un programme proposé basé sur l'auto-apprentissage pour le développement des compétences de la lecture, de l'écriture et de l'autonomie chez les futurs enseignants à la section de français à la faculté de pédagogie.Thèse de doctorat. Faculté de pédagogie. Université d'Ain-Chams.

28. Robert, J., P. (2002). Dictionnaire pratique de didactique du FLE. Paris : Ophrys .

29. Sabri, N. (2009). Efficacité d'un programme propose à la lueur de l'Approche des histoires sur le développement de quelques compétences de la compréhension lecturale en langue française et de valeurs morales chez les élèves du cycle préparatoire, Thèse de doctorat. Faculté de Jeunes Filles. Université d'Ain Chams.

30. Sobhi, W. (2010). Effet de la stratégie du brainstorming sur le développement des compétences de la lecture critique chez les étudiants du département de français aux facultés des pédagogies. Thèse de Magistère. Faculté de Pédagogie. Université de Ménoufya.

31. Soleiman, R. (1996). Efficacité du programme proposé pour le développement de certaines compétences auditives chez les étudiants des facultés de pédagogies section de français. Thèse de Magistère. Faculté de pédagogie. Université de Mansourah.

32. Turcotte, C. (1992). Un cadre théorique sur la lecture et un modèle du lecteur (conçus en vue de l'évaluation diagnostique du lecteur cégépien). Mémoire de maîtrise. Université de Sherbrooke.

33. Vacca, R.T. et Vacca, A.L. (2002). Content area reading: Literacy and learning across the Curriculum, (7e éd.). Boston: Allyn and Bacon.

34. Weiner, H.S. et Bazerman C. (1997). Reading textbooks: A college skills handbook. Boston: Houghton Mifflin Company.

\section{Sitograghies}

35. http://heritage.csdecou.qc.ca/beaulieum/resume

36. https://www.k12.gov.sk.ca/docs/francais/fransk/fran/sec/prg_etudes/str atl13.html 